Abstract OC-116 Table 1 Colonoscopy outcomes

\begin{tabular}{lcccrrr}
\hline Colonoscopy outcome & $\begin{array}{l}\text { FOB } \\
\text { numbers }\end{array}$ & $\begin{array}{l}\text { FIT } \\
\text { numbers }\end{array}$ & $\begin{array}{l}\text { Total } \\
\text { numbers }\end{array}$ & FOB\% & FIT\% & Total\% \\
\hline Unknown & 40 & 204 & 244 & 6.6 & 4.4 & 4.7 \\
Routine recall & 229 & 2582 & 2811 & 37.6 & 55.9 & 53.7 \\
Surveillance-intermediate & 119 & 877 & 996 & 19.5 & 19.0 & 19.0 \\
risk & & & & & & \\
Surveillance-high risk & 70 & 466 & 536 & 11.5 & 10.1 & 10.2 \\
Diagnosed with IBD & 22 & 98 & 120 & 3.6 & 2.1 & 2.3 \\
Diagnosed with cancer & 120 & 294 & 414 & 19.7 & 6.4 & 7.9 \\
Ceased (other reasons) & 2 & 12 & 14 & 0.3 & 0.3 & 0.3 \\
Repeat procedure needed & 7 & 88 & 95 & 1.1 & 1.9 & 1.8 \\
Total & 609 & 4621 & 5230 & 100.0 & 100.0 & 100.0 \\
\hline
\end{tabular}

Conclusion The first round of screening was very successful demonstrating a pathology yield of over $70 \%$. BSW face ongoing challenges with interesting new developments for a maturing programme. BSW is now well placed to begin planning further age expansion and development.

Competing interests None declared.

\section{OC-117 NEOADJUVANT PRECISION CHEMOEMBOLISATION FOR EASILY RESECTABLE COLORECTAL LIVER METASTASES}

doi:10.1136/gutjnl-2012-302514a.117

R Jones,* D Dunne, S W Fenwick, P Sutton, H Malik, G Poston. Department of Hepatobiliary Surgery, Aintree University Hospital NHS Foundation Trust, Liverpool, UK

Introduction Peri-operative chemotherapy confers a 3-year progression free survival advantage for patients with colorectal liver metastases. Degree of post-chemotherapy tumour necrosis is associated with disease free survival. However, systemic neoadjuvant chemotherapy is associated with pathological damage to hepatic parenchyma, increasing perioperative morbidity and mortality. Irinotecan eluting beads (DEBIRI-TACE) are delivered to tumour intra-arterially, where they provide controlled \& sustained delivery of Irinotecan directly to tumour, maximising response and reducing systemic exposure. This study aimed to examine the feasibility and safety of a single neoadjuvant bead embolisation 1-month before hepatectomy.

Methods Patients with easily resectable colorectal liver metastases received DEBIRI-TACE 1 month before surgery. Primary end-point was tumour resectability, Secondary end points included pathological tumour response and safety.

Results TACE attempted in 49 patients and was successful in 40 . Reasons for failed TACE included arterial abnormality $(n=2)$, progressive disease $(n=2)$, bilobar disease $(n=2)$, hepatoma $(n=1)$, allergy to contrast $(n=1)$ and concomitant infection $(n=1)$. There was one post-TACE liver abscess (3\%), and 1 post TACE pancreatitis (3\%) (recognised complications). 38 patients have undergone hepatic resection so far, with $\mathrm{R} 0$ resection rate of $100 \%$ and no significant post-hepatectomy morbidity. Thirty day post-operative mortality was $7.6 \%(n=2)$, with neither death related to TACE (one intraoperative pneumomediastinum, one MODS after aspiration pneumonia). Complete pathological response (no viable tumour) was demonstrated in $15 \%$ of lesions, major response in $55 \%$ and minor response in $30 \%$.

Conclusion Neoadjuvant DEBIRI TACE for resectable colorectal liver metastasis is safe and is not associated with increased post-hepatectomy morbidity. A single treatment with DEBIRI-TACE resulted in pathological response of tumour similar to that seen after systemic treatment, which may translate to improved progression free survival.

Competing interests None declared.
EXTRAMURAL VASCULAR INVASION (EMVI) IS A BETTER PROGNOSTIC INDICATOR IN PT4 COLORECTAL CANCER THAN PATHOLOGICAL SUBTYPING INTO PT4A AND PT4B: CLINICOPATHOLOGICAL ANALYSIS OF 276 CASES

doi:10.1136/gutjnl-2012-302514a.118

R Sreekumar, ${ }^{*}$ A Mirnezami, R Turck, M Bullock, T Cheung, A Bruce. Cancer Sciences Division, Southampton general hospital, Southampton, UK

Introduction The presence of extramural vascular invasion (EMVI) has been associated with reduced survival in colorectal cancer (CRC), and failure to consider it may account for discrepancies in outcome between similar stages. One clinically and pathologically heterogeneous subtype of CRC is T4 disease. At the microscopic level, some tumours are classed as $\mathbf{p T} \mathbf{T a}$ due to invasion of local organs, while others are defined as $\mathbf{p} \mathbf{T} \mathbf{4} \mathbf{b}$ due to invasion of the visceral peritoneum (based on 5th edition of TNM). The aim of the present study was to compare $\mathrm{T} 4 \mathrm{a}$ and $\mathrm{T} 4 \mathrm{~b}$ colorectal cancers for EMVI status and longterm outcome.

Methods Pathological data on consecutive cases of T4 colorectal cancer proceeding to surgery were extracted from a prospectively collected database between 2004 and 2011. Pathological parameters analysed included macroscopic tumour details, differentiation, nodal status, and the presence of EMVI. Patient demographics, disease stage, and longterm oncological outcomes were evaluated in all cases.

Results 276 consecutive cases of T4 colorectal cancer were identified during the study period. $92 \%$ of tumours were colonic and $8 \%$ rectal. $79 \%$ of tumours were $\mathrm{T} 4 \mathrm{~b}$, and the remainder $\mathrm{T} 4 \mathrm{a} .35 \%$ of cases were stage II disease, $43 \%$ stage III, and $22 \%$ stage IV. No difference was noted between $\mathrm{T} 4 \mathrm{a}$ and $\mathrm{T} 4 \mathrm{~b}$ tumours for tumour differentiation, or lymph node positivity. No difference in cancer specific and disease free survival were noted between pT4a and pT4b tumours, however significantly divergent survival curves were found for EMVI positive and negative disease. The median cancer specific survival for T4a vs T4b was 32 months vs 41 months respectively $(\log$ rank $\mathbf{p}=\mathbf{0 . 5 6 9})$. Median disease free survival for the same cohort was 23 months vs 36 months respectively (log rank $\mathbf{p}=\mathbf{0 . 8 8 2}$ ). Median Cancer specific survival in patients with and without EMVI was 25 vs 60 months respectively (log rank p. Median disease free survival was $\mathbf{1 5}$ months for those with EMVI, compared to 64 months in those without (log rank p. Conclusion Subtyping of T4 tumours by EMVI status may be a better prognostic indicator than division into $\mathrm{T} 4 \mathrm{a}$ and $\mathrm{T} 4 \mathrm{~b}$.

Competing interests None declared.

\section{OC-119 MECHANISTIC RANDOMISED CONTROL TRIAL OF MESALAZINE IN SYMPTOMATIC DIVERTICULAR DISEASE}

doi:10.1136/gutjnl-2012-302514a.119

${ }^{1,2} \mathrm{~J}$ Smith, ${ }^{* 2} \mathrm{D}$ Humes, ${ }^{1} \mathrm{~K}$ Garsed, ${ }^{1} \mathrm{C}$ Lam, ${ }^{3} \mathrm{~A}$ Zaitoun, ${ }^{4} \mathrm{~A}$ Bennett, ${ }^{2} \mathrm{~J}$ Scholefield, ${ }^{1} \mathrm{R}$ Spiller. ${ }^{1}$ NIHR Biomedical Research Unit, Nottingham, UK; ${ }^{2}$ Department of Surgery, Nottingham, UK; ${ }^{3}$ Department of Pathology, University Hospitals Nottingham, Nottingham, UK; ${ }^{4}$ FRAME Laboratories, University of Nottingham, Nottingham, UK

Introduction Painful symptomatic diverticular disease (SDD) is a common but poorly understood condition. Approximately 20\% of patients with diverticulosis complain of pain, but there are currently no effective treatments. We have previously reported peripheral immune activation and alteration in colonic nerve function in SDD. Aims To test the significance of this immune activation by performing the first parallel design, double blind, randomised placebo controlled trial of an anti-inflammatory drug, mesalazine in SDD. 